\title{
ANTICHAINS AND PRODUCTS IN PARTIALLY ORDERED SPACES $\left({ }^{1}\right)$
}

\author{
BY \\ E. D. TYMCHATYN
}

1. Introduction. By a partially ordered space $X$ we mean a Hausdorff space $X$ with a partial order which is closed when regarded as a subset of $X \times X$. In this paper $X$ will denote a compact partially ordered space. The symbol $2^{X}$ will denote the space of compact subsets of $X$ with the finite topology [3]. This topology has as a basis all finite tuples $\left\langle V_{1}, \ldots, V_{n}\right\rangle$ where each $V_{i}$ is an open subset of $X$. A closed subset $A$ of $X$ is in $\left\langle V_{1}, \ldots, V_{n}\right\rangle$ if $A$ meets $V_{i}$ for each $i$ and if $A \subset V_{1} \cup \ldots$ $\cup V_{n}$. The space $2^{X}$ is compact and Hausdorff. If $X$ is metric then $2^{X}$ is metric and the finite topology on $2^{x}$ is the topology induced by the Hausdorff metric.

For each $x \in X$ we let

$$
L(x)=\{y \in X \mid y \leqq x\}, \quad M(x)=\{y \in X \mid x \leqq y\}
$$

and $\Gamma(x)=L(x) \cup M(x)$. If $A \subset X$ we let $L(A)=\bigcup\{L(x) \mid x \in A\}$. We define $M(A)$ and $\Gamma(A)$ similarly. We let $L=\{x \in X \mid L(x)=\{x\}\}$ and we let

$$
M=\{x \in X \mid M(x)=\{x\} \text {. }
$$

We call $L$ (resp. $M$ ) the set of minimal (resp. maximal) elements of $X$.

A chain is a totally ordered set. An order arc is a compact and connected chain. It is easy to check that a nondegenerate and separable order arc is homeomorphic to the unit interval. An antichain is a totally unordered set. It is known [5] that each chain is contained in a maximal chain. Each maximal chain is a closed set which meets both $L$ and $M$. Thus $L$ and $M$ are maximal antichains.

Let $X$ be a compact metric partially ordered space such that for each $x \in X$ $\Gamma(x)$ is connected. We prove that the family of compact maximal antichains of $X$ covers $X$ if and only if $L$ and $M$ are closed. The family of compact maximal antichains of $X$ admits the structure of a topological lattice. If $L$ and $M$ are closed and the maximal chains of $X$ are pairwise disjoint order arcs we prove that $X$ is homeomorphic to $M \times[0,1]$ (the Cartesian product). We study the role of those partially ordered spaces in which the maximal chains are pairwise disjoint. In $\$ 5$ we use these results to get a characterization of the 2-cell.

We shall need the following theorem which is due to R. J. Koch. This theorem also appears in [6].

Received by the editors May 6, 1969.

(1) This research was supported in part by a grant from the National Science Foundation.

Copyright $\mathbb{C}$ 1970, American Mathematical Society 
THEOREM 1.1 (KOCH). If $X$ is a compact partially ordered space such that $L$ is closed and for each $x \in X, L(x)$ is connected, then each maximal order arc in $X$ meets $L$.

Definition 1.2. If $X$ is a compact partially ordered space let $\mathscr{M}(X)$ denote the set of those order arcs in $X$ which meet both $L$ and $M$. We consider $\mathscr{M}(X)$ as a subspace of $2^{X}$.

The next three lemmas appear in [7].

LEMMA 1.3. If $X$ is a compact partially ordered space then the family of compact chains of $X$ is a compact subset of $2^{X}$.

LEMMA 1.4. Let $X$ be a compact partially ordered space. If there exists a family $\mathscr{C}$ of maximal chains of $X$ such that $X \subset \cup \mathscr{C}$ and $\mathscr{C}$ is a compact subset of $2^{X}$ then $L$ and $M$ are compact.

LEMMA 1.5. If $X$ is a compact partially ordered space such that $L$ and $M$ are closed then $\mathscr{M}(X)$ is a compact subset of $2^{X}$.

2. The product theorem. We let $[0,1]$ denote the unit interval with its usual order.

Definition 2.1. If $Y$ is a compact Hausdorff space we let $P(Y)$ denote the space $Y \times[0,1]$ with the product topology and with the partial order $(y, a) \leqq(x, b)$ if and only if $y=x$ and $a \leqq b$. Then $P(Y)$ is a compact partially ordered space.

LEMMA 2.2. If $X$ is a compact partially ordered space such that $L$ is closed and for each $x \in X, L(x)$ has a unique minimal element then $L$ is a retract of $X$.

Proof. For each $x \in X$ let $\pi(x)$ denote the unique minimal element of $L(x)$. This defines a function $\pi: X \rightarrow L$ such that $\pi$ leaves every element of $L$ fixed.

We must show that $\pi$ is continuous. Let $x \in X$ and let $x_{i}$ be a net converging to $x$. Let $z$ be a cluster point of the net $\pi\left(x_{i}\right)$. Then $z \in L(x)$ since the partial order on $X$ is closed and $z \in L$ since $L$ is closed. Hence $z=\pi(x)$ and $\pi$ is continuous.

DefinItION 2.3. A metric $r$ for a partially ordered space $X$ is radially convex if $x \leqq y \leqq z$ implies $r(x, y)+r(y, z)=r(x, z)$.

The basic theorem about radially convex metrics is due to J. H. Carruth [1].

THEOREM 2.4. Every compact, metric, partially ordered space $X$ admits a radially convex metric.

LEMMA 2.5. Let $X$ be a compact partially ordered space which contains a compact maximal antichain $C$. If $T$ is an order arc which meets both $L$ and $M$ then $T$ meets $C$.

Proof. Let $P=\{x \in T \mid x \in L(C)\}$ and let $Q=\{x \in T \mid x \in M(C)\}$. Then $P$ and $Q$ are closed. Since $C$ is a maximal antichain $P \cup Q=T$. Now $T \cap L \subset P$ and $T \cap M \subset Q$. Since $T$ is connected $P \cap Q$ is nonvoid. If $z \in P \cap Q$ then $z \in C$ since $C$ is an antichain. 
THEOREM 2.6. Let $X$ be a compact metric partially ordered space such that $L$ and $M$ are compact subsets of $X$ and for each $x \in X, \Gamma(x)$ is connected. Then there exists a continuous one-to-one function $f:[0,1] \rightarrow 2^{X}$ such that

(i) for each $a \in[0,1] f(a)$ is a compact maximal antichain of $X$,

(ii) if $a<b$ in $[0,1]$ then $f(a) \subset L(f(b))$,

(iii) $f(0)=L$ and $f(1)=M$,

(iv) $X \subset \bigcup\{f(a) \mid a \in[0,1]\}$,

(v) if $M \cap L$ is void and if $a<b$ in $[0,1]$ then $f(a) \cap f(b)$ is void.

\section{Proof.}

Case 1. Suppose $M \cap L$ is void. Let $Y$ be the compact Hausdorff space obtained from $X$ by pinching $L$ and $M$ to points [2, p. 98]. Let $\pi$ be the projection of $X$ onto $Y$. Define a partial order on $Y$ by setting $x \leqq y$ in $Y$ if and only if there exist $x^{\prime} \in \pi^{-1}(x)$ and $y^{\prime} \in \pi^{-1}(y)$ with $x^{\prime} \leqq y^{\prime}$. Then $Y$ is a compact metric partially ordered space with unique minimal element $\pi(L)$ and unique maximal element $\pi(M)$.

By Theorem 2.4, $Y$ admits a radially convex metric $r$ such that $r(\pi(L), \pi(M))=1$. For each $a \in[0,1]$ let $B(a)=\{y \in Y \mid r(\pi(L), y)=a\}$ and let $f(a)=\pi^{-1}(B(a))$.

Since $r$ is a radially convex metric $B(a)$ is an antichain in $Y$ and $f(a)$ is an antichain in $X$. By Koch's theorem $f(a)$ is a maximal antichain. Since $B(a)$ is compact and $\pi$ is continuous, $f(a)=\pi^{-1}(B(a))$ is compact. Everything else is clear except that $f$ is continuous.

Let $a_{i}$ ) be a sequence which converges to $a$ in $[0,1]$. Let $\left.x_{i}\right)$ be a sequence which converges to $x$ in $X$ such that for each $i, x_{i} \in f\left(a_{i}\right)$. Since $\pi$ is continuous

$$
r(\pi(x), \pi(L))=\lim r\left(\pi\left(x_{i}\right), \pi(L)\right)=\lim a_{i}=a .
$$

Hence $\pi(x) \in B(a)$ and $x \in f(a)$. Thus each cluster point in $2^{x}$ of the sequence $\left.f\left(a_{i}\right)\right)$ is contained in $f(a)$. Let $x \in f(a)$. By Koch's theorem there exists an order arc $T$ such that $x \in T$ and $T$ meets both $L$ and $M$. For each $i, f\left(a_{i}\right)$ meets $T$ by Lemma 2.5. For each $i$ let $y_{i} \in T \cap f\left(a_{i}\right)$ and let $y$ be a cluster point of the sequence $\left.y_{i}\right)$. Since $T$ is closed $y \in T$. By the above argument $y \in f(a)$. Since $f(a)$ is an antichain $y=x$. Thus, the sequence $f\left(a_{i}\right)$ ) converges to $f(a)$ in $2^{x}$ and $f$ is continuous.

Case 2. Suppose $M \cap L$ is nonvoid. Make the disjoint union of $P(L)$ and $X$ into a partially ordered space by extending the partial orders on $P(L)$ and $X$ so that if $(n, a) \in P(L)$ and $x \in X$ then $(n, a) \leqq x$ if and only if $n \leqq x$ in $X$. Form the adjunction space $X^{\prime}$ of $P(L)$ and $X$ by identifying $(n, 1)$ with $n$ for each $n \in L$. The partial order on $X \cup P(L)$ induces a partial order $\leqq$ on $X^{\prime}$ such that $\left(X^{\prime}, \leqq \prime\right)$ satisfies all the hypotheses of the theorem. The set of minimal (resp. maximal) elements of $X^{\prime}$ is $L \times\{0\}$ (resp. $M$ ). We may apply Case 1 to the partially ordered space $X^{\prime}$ to obtain a continuous one-to-one function $f^{\prime}:[0,1] \rightarrow 2^{X^{\prime}}$ such that conditions (i)-(v) are satisfied. For each $a \in[0,1]$ let

$$
f^{\prime \prime}(a)=\left\{x \in f^{\prime}(a) \cup L \mid x \text { is maximal in } f^{\prime}(a) \cup L\right\} .
$$


Then $f^{\prime \prime}(a)$ is a compact maximal antichain. Using $f^{\prime \prime}$ it is easy to define a continuous one-to-one function $f:[0,1] \rightarrow 2^{x}$ such that conditions (i)-(iv) are satisfied.

COROLlaRY 2.7. Let $X$ be a compact metric partially ordered space such that $L$ and $M$ are closed subsets of $X$ and for each $x \in X, \Gamma(x)$ is a nondegenerate order arc. Then $X$ is homeomorphic under an order preserving map to $P(M)$.

Proof. Let $f:[0,1] \rightarrow 2^{x}$ be a one-to-one continuous function such that conditions (i)-(v) of Theorem 2.6 are satisfied. Define $h: P(M) \rightarrow X$ by letting $h(m, a) \in L(m) \cap f(a)$ for each $(m, a) \in P(M)=M \times[0,1]$.

Let $(m, a) \in M \times[0,1]$ and let $\left.\left(m_{i}, a_{i}\right)\right)$ be a sequence converging to $(m, a)$. Let $z$ be a cluster point of $\left.h\left(m_{i}, a_{i}\right)\right)$. The sequence $\left.m_{i}\right)$ converges to $m$ in $X$. For each $i$ $h\left(m_{i}, a_{i}\right) \in L\left(m_{i}\right)$. Since the partial order on $X$ is closed $z \in L(m)$. Since the sequence $f\left(a_{i}\right)$ converges to $f(a)$ in $2^{X}, z \in f(a)$. Thus $z=h(m, a)$ and $f$ is continuous. By conditions (iv), (ii) and (v) of Theorem $2.6 h$ is onto $X$, one-to-one and order preserving. Since $P(M)$ is compact and $X$ is Hausdorff, $h$ is a homeomorphism.

The hypotheses of Corollary 2.7 may be relaxed as follows:

THEOREM 2.8. Let $X$ be a compact metric partially ordered space such that for each $x \in X, \Gamma(x)$ is a chain. If $X$ contains a compact maximal antichain $A$ then $X$ may be embedded by an order preserving map into $P(A)$.

Proof. By Theorem 2.4 there exists a radially convex metric $r$ for $X$ such that the diameter of $X$ with respect to $r$ is less than $1 / 2$.

Define $g: X \rightarrow A$ by letting $g(x) \in \Gamma(x) \cap A$ for each $x \in X$. By Lemma 2.2 $g$ is a retraction of $X$ onto $A$.

Define $f: X \rightarrow P(A)$ as follows: For $x \in X$ let

$$
\begin{array}{ll}
f(x)=(g(x), 1 / 2+r(x, g(x))) & \text { if } g(x) \leqq x \text { and } \\
f(x)=(g(x), 1 / 2-r(x, g(x))) & \text { if } x \leqq g(x) .
\end{array}
$$

Since $g$ is continuous and $r$ is a metric for $X, f$ is continuous. Because $r$ is radially convex $f$ is one-to-one and order preserving. Since $X$ is compact and $P(A)$ is Hausdorff, $f$ is open.

The following theorem is the main result in this paper.

THEOREM 2.9. Let $X$ be a compact metric partially ordered space such that $L$ and $M$ are closed and disjoint and for each $x \in X, \Gamma(x)$ is connected. There exists an order preserving map $g: P(\mathscr{M}(X)) \rightarrow X$ such that

(a) for each $T \in \mathscr{M}(X), g$ maps the maximal chain $\{T\} \times[0,1]$ of $P(\mathscr{M}(X))$ homeomorphically onto the maximal chain $T$ of $X$,

(b) for each $y \in[0,1], g(\mathscr{M}(X) \times\{y\})$ is a compact maximal antichain of $X$, and

(c) the function $f:[0,1] \rightarrow 2^{X}$ defined by letting $f(y)=g(\mathscr{M}(X) \times\{y\})$ satisfies conditions (i)-(v) of Theorem 2.6. 
Proof. Let $f:[0,1] \rightarrow 2^{x}$ be a function which satisfies conditions (i)-(v) of Theorem 2.6. By Lemma 1.5, $\mathscr{M}(X)$ is a compact subset of $2^{X}$. Define

$$
g: P(\mathscr{M}(X)) \rightarrow X
$$

by letting $g(T, a)$ be the unique element in $T \cap f(a)$ for each $(T, a) \in P(\mathscr{M}(X))$.

Let $\left.\left(T_{i}, a_{i}\right)\right)$ be a net in $P(\mathscr{M}(X))$ which converges to $(T, a)$ and let $z$ be a cluster point of the net $g\left(T_{i}, a_{i}\right)$ ). Since the nets $\left.T_{i}\right)$ and $\left.f\left(a_{i}\right)\right)$ converge in $2^{X}$ to $T$ and $f(a)$ respectively, $z \in T \cap f(a)$ and $g$ is continuous. Everything else is now clear from the definition of $g$.

We can prove analogous theorems for much more general situations.

THEOREM 2.10. Let $X$ be a compact partially ordered space and let $\mathscr{C}$ be a compact family (in $2^{X}$ ) of closed chains of $X$ such that $X \subset \cup \mathscr{C}$. Let $g: Y \rightarrow \mathscr{C}$ be a continuous function from a compact Hausdorff space $Y$ onto $\mathscr{C}$. There exists a compact partially ordered space

$$
Y^{*}=\{(y, z) \mid y \in Y \text { and } z \in g(y)\}
$$

and an order preserving map $f$ of $Y^{*}$ onto $X$ such that

(i) the maximal chains of $Y^{*}$ are pairwise disjoint,

(ii) $Y$ is homeomorphic to both the set of maximal elements of $Y^{*}$ and to the set of minimal elements of $Y^{*}$, and

(iii) for each $y \in Y$, $f$ maps the maximal chain $\{(y, z) \mid z \in g(y)\}$ of $Y^{*}$ homeomorphically onto the chain $g(y)$ in $X$.

Theorem 2.10 is useful where $\mathscr{C}$ can be taken to be a subset of $\mathscr{M}(X)$. If $\mathscr{C}$ has a topological property $P$ then one may be able to prove that $Y^{*}$ and hence $X$ has property $P$. Local connectedness and connectedness are two such properties. We shall give some more detailed examples in the next section.

Proof of 2.10. Let the set $Y \times X$ have the product topology and the partial order $(a, b) \leqq(c, d)$ if and only if $a=c$ and $b \leqq d$. Then $Y \times X$ is a compact partially ordered space. Let $Y^{*}=\{(y, z) \in Y \times X \mid z \in g(y)\}$.

Let $\left.\left(y_{i}, z_{i}\right)\right)$ be a net in $Y^{*}$ which converges to $(y, z)$. Then the net $\left.y_{i}\right)$ converges to $y$ in $Y$ and the net $z_{i}$ ) converges to $z$ in $X$. Since $g$ is continuous the net $g\left(y_{i}\right)$ ) converges to $g(y)$ in $2^{x}$. Hence $z \in g(y)$ and $(y, z) \in Y^{*}$. Thus $Y^{*}$ with the relative topology and the partial order inherited from $Y \times X$ is a compact partially ordered space.

The maximal chains in $Y^{*}$ are the sets $\{(y, z) \mid z \in g(y)\}$ where $y \in Y$. The set of maximal elements of $Y^{*}$ is

$$
\{(y, z) \mid y \in Y \text { and } z \text { is the maximal element of } g(y)\} .
$$

Let $\left.\left(y_{i}, z_{i}\right)\right)$ be a net of maximal elements of $Y^{*}$ which converges to $(y, z)$. As above, the net $\left.g\left(y_{i}\right)\right)$ converges to $g(y)$ in $2^{X}$ and the net $z_{i}$ ) converges to $z$ in $X$. For each $i, g\left(y_{i}\right) \subset L\left(z_{i}\right)$. Since the partial order on $X$ is closed $g(y) \subset L(z)$. Since 
$z \in g(y), z$ is the maximal element of $g(y)$ in $X$. Hence, $(y, z)$ is a maximal element of $Y^{*}$ and the set of maximal elements of $Y^{*}$ is compact.

Define a function $f: Y^{*} \rightarrow X$ by letting $f(y, z)=z$ for each $(y, z) \in Y^{*}$. Then, $f$ is continuous since $f$ is the restriction to $Y^{*}$ of the natural projection of $Y \times X$ onto $X$. The rest is now clear.

3. L-continuity. The partial order on $X$ is said to be $L$-continuous if the function $f: X \rightarrow 2^{x}$ defined by $f(x)=L(x)$ is continuous.

LEMMA 3.1 (WARD [6]). If the partial order on a compact partially ordered space $X$ is $L$-continuous then $L$ is closed.

We prove a partial converse to Lemma 3.1.

Proposition 3.2. Let $X$ be a compact partially ordered space. If $L$ is closed and for each $x \in X, L(x)$ is an order arc, then the partial order on $X$ is L-continuous.

Proof. Let $x_{i}$ ) be a net which converges to $x$ in $X$. Since $2^{x}$ is compact the net $\left.L\left(x_{i}\right)\right)$ has a cluster point $A$ in $2^{x}$. Since the partial order on $X$ is closed $A \subset L(x)$. By a result in [3] $A$ is connected. Clearly $x \in A$. For each $i, L\left(x_{i}\right) \cap L$ is nonvoid and $L$ is closed. It follows that $A$ meets $L$. Since $L(x)$ is a continuum which is irreducible with respect to containing $x$ and $L(x) \cap L, A=L(x)$. Thus the partial order on $X$ is $L$-continuous.

THEOREM 3.3. Let $X$ be a compact partially ordered space such that for each $x \in X, L(x)$ is a chain. Then (i) is equivalent to (ii) and (iii) implies (i):

(i) the family $\mathscr{C}$ of maximal chains of $X$ is closed in $2^{X}$,

(ii) $M$ is closed and the partial order on $X$ is L-continuous,

(iii) the family of compact maximal antichains of $X$ covers $X$.

Proof. By Lemma 1.4 and Lemma 3.1, $L$ and $M$ are closed if either (i) or (ii) holds.

(i) implies (ii). Let $x_{i}$ ) be a net converging to $x$ in $X$. For each $i$, let $m_{i} \in M \cap M\left(x_{i}\right)$ and let $m$ be a cluster point of $m_{i}$ ). Then $m \in M \cap M(x)$. We may suppose $m_{i}$ ) converges to $m$. Since $\mathscr{C}$ is compact and the partial order on $X$ is closed $L\left(m_{i}\right)$ converges to $L(m)$. Hence $\left.L\left(x_{i}\right)\right)$ converges to $L(x)$ and the partial order on $X$ is $L$-continuous.

(ii) implies (i). Define $f: M \rightarrow \mathscr{C}$ by letting $f(m)=L(m)$ for each $m \in M$. Since the partial order on $X$ is $L$-continuous, $f$ is a homeomorphism of the compact space $M$ onto $\mathscr{C}$. Thus $\mathscr{C}$ is compact.

(iii) implies (i). Let $C_{i}$ ) be a net of members of $\mathscr{C}$ which converges to some $C \in 2^{X}$. By Lemma 1.3, $C$ is a chain. Let $m \in M$ such that $C \subset L(m)$ and let $x \in L(m)$. Let $A$ be a compact maximal antichain which contains $x$. For each $i, M(A) \cap C_{i}$ $\cap M$ is nonvoid. Since $L\left(C_{i} \cap M\right)=C_{i}$ is a chain $C_{i} \cap A$ is nonvoid. For each $i$ let $a_{i} \in C_{i} \cap A$. Each cluster point of $\left.a_{i}\right)$ is in $A \cap C$. Now $A \cap C \subset L(m) \cap A$. 
Since $x \in L(m) \cap A, A$ is an antichain and $L(m)$ is a chain it follows that $a_{i}$ ) converges to $x$. Thus $L(m)=C$ and $C \in \mathscr{C}$. Hence $\mathscr{C}$ is compact.

Proposition 3.4. Let $X$ be a compact partially ordered space such that $L$ and $M$ are closed and for each $x \in X, \Gamma(x)$ is an order arc. Define $\pi: X \rightarrow M$ by letting $\pi(x) \in M \cap M(x)$ for each $x \in X$. Then $\pi$ is continuous and open.

Proof. By Lemma 3.2, $\pi$ is continuous.

Let $x \in X$ and let $U$ be a neighbourhood of $x$. Then $\langle U, X\rangle$ is a neighbourhood of $\Gamma(x)$ in $2^{x}$. By Theorem 3.2 there exists a neighbourhood $V$ of $\pi(x)$ such that $\Gamma(V) \subset\langle U, X\rangle$. Then $V \cap M$ is a neighbourhood of $\pi(x)$ in $M$ such that $V \cap M$ $\subset \pi(U)$. Thus $\pi$ is open.

Proposition 3.5. Let $X$ be a compact partially ordered space such that for each $x \in X, \Gamma(x)$ is a real order arc. If $L$ and $M$ are compact and first countable (resp. separable) then $X$ is first countable (resp. separable).

Proof. Use Theorem 3.2 and a result of Nachbin [4, p. 48].

In Theorem 2.6 we gave sufficient conditions for a metric partially ordered space to be covered by compact maximal antichains. We would like to extend this result to nonmetric partially ordered spaces.

Problem 1. Let $X$ be a compact partially ordered space such that $L$ and $M$ are closed and for each $x \in X, \Gamma(x)$ is a nondegenerate order arc. Does each point of $X$ lie in a compact maximal antichain?

Problem 2. Let $X$ be a compact partially ordered space such that $L$ and $M$ are compact metric spaces. Is $X$ metric if, for each $x \in X, \Gamma(x)$ is a separable order arc? In view of Theorem 2.10 it suffices to consider the case where $M$ is homeomorphic to Cantor's ternary set.

The answer to Problem 2 is affirmative in case there exists a family. $\mathscr{F}$ of compact maximal antichains such that $X \subset \bigcup \mathscr{F}$ and $\mathscr{F}$ is a compact subset of $2^{X}$. L. Mohler and L. E. Ward, Jr. suggested the following example. It shows that the conditions on $X$ can not be relaxed.

EXAmple 3.6. Let $X$ be the set $[0,1] \times[0,1]$ with the topology of the comb space. A basic open neighbourhood of $(x, 0)$ has the form

$$
(U \times[0,1]) \backslash \bigcup_{i=1}^{n}\left(\left\{x_{i}\right\} \times\left[y_{i}, z_{i}\right]\right)
$$

where $U$ is a neighbourhood of $x$ in $[0,1], x_{i} \in U, 0<y_{i} \leqq z_{i} \leqq 1$ and $n$ is a nonnegative integer. If $0<y \leqq 1$ a basic open neighbourhood of $(x, y)$ has the form $\{x\} \times V$ where $V$ is a neighbourhood of $y$ in $[0,1]$. Give $X$ the partial order $(m, x)$ $\leqq(n, y)$ if and only if $m=n$ and $x \leqq y$. Then $X$ is a compact partially ordered space such that $L=[0,1] \times\{0\}$ and $M=[0,1] \times\{1\}$. Notice that $X$ has all the properties of the space in Problem 2 except that $M$ is not closed. However, $X$ is not separable. 
4. Compact maximal antichains. We shall define lattice operations on the space of compact maximal antichains of a compact partially ordered space $X$.

Definition 4.1. A topological lattice is a Hausdorff space $X$ with two continuous, associative, commutative and idempotent operations $\vee$ and $\wedge$ such that for $x, y \in X, x \vee(y \wedge x)=x \wedge(y \vee x)=x$ and the conditions $x \wedge y=y$ and $x \vee y=x$ are equivalent.

Definition 4.2. If $X$ is a compact partially ordered space let $\mathscr{A}(X)$ denote the space of compact maximal antichains of $X$ with its relative topology as a subset of $2^{X}$. For $C, D \in \mathscr{A}(X)$ let

$$
C \vee D=\{x \in C \cup D \mid x \text { is maximal in } C \cup D\}
$$

and let

$$
C \wedge D=\{x \in C \cup D \mid x \text { is minimal in } C \cup D\} .
$$

LEMMA 4.3. Let $X$ be a compact partially ordered space. If $C, D \in \mathscr{A}(X)$ then $C \vee D$ and $C \wedge D$ are compact antichains.

Proof. Clearly $C \vee D$ and $C \wedge D$ are antichains. Let $x_{i}$ ) be a net in $C \vee D$ which converges to $x \in X$. We may suppose that for each $i, x_{i} \in C$. Since $C$ is closed $x \in C$. For each $i$, let $y_{i} \in D \cap L\left(x_{i}\right)$ and let $y$ be a cluster point of $\left.y_{i}\right)$. Then $y \in D \cap L(x)$. Since $C$ and $D$ are antichains $x$ is maximal in $C \cup D$. Thus $x \in C \vee D$ and $C \vee D$ is closed. Similarly $C \wedge D$ is closed.

LEMMA 4.4. Let $X$ be a compact partially ordered space such that $L$ and $M$ are closed and for each $x \in X, \Gamma(x)$ is connected. If $C, D \in \mathscr{A}(X)$ then $C \vee D$ and $C \wedge D$ are in $\mathscr{A}(X)$.

Proof. Let $x \in X$. By Koch's theorem there exists an order arc $T$ such that $x \in T$ and $T$ meets both $L$ and $M$. By Lemma 2.5 there exists $y \in T \cap C$ and $z \in T \cap D$. Since $T$ is a chain $x \in \Gamma(C \vee D) \cap \Gamma(C \wedge D)$. By Lemma 4.3, $C \wedge D$ and $C \vee D$ are antichains hence $C \wedge D$ and $C \vee D$ are in $\mathscr{A}(X)$.

THEOREM 4.5. Let $X$ be a compact partially ordered space such that $L$ and $M$ are closed and for each $x \in X, \Gamma(x)$ is connected. Then $\mathscr{A}(X)$ with operations $\wedge$ and $\vee$ is a topological lattice with minimal element $L$ and maximal element $M$. If $X$ is metric then $\mathscr{A}(X)$ is arcwise connected.

Proof. By Lemma $4.4 \wedge$ and $\vee$ are operations on $\mathscr{A}(X)$. The proof that $\vee$ and $\wedge$ are associative operations is similar to the proof of Lemma 4.4.

Let $C_{i}$ ) and $D_{i}$ ) be nets in $\mathscr{A}(X)$ which converge to $C$ and $D$ respectively. Let $H$ be a cluster point of the net $C_{i} \vee D_{i}$ ) in $2^{x}$ and let $x \in H$. For each $i$ let $x_{i} \in C_{i} \vee D_{i}$ such that $x_{i}$ ) converges to $x$. Then $x \in C \cup D$. If $x \in C \cap D$ then $x \in C \vee D$. If $x \in D \backslash C$ then eventually $x_{i} \in D_{i} \mid C_{i}$. Thus $x_{i} \in M\left(C_{i}\right)$ eventually. Since $\left.C_{i}\right)$ converges to $C$ and the partial order on $X$ is closed $x \in M(C)$. Thus $x \in C \vee D$ and $H \subset C \vee D$. For each $i$ and each maximal order $\operatorname{arc} T\left(C_{i} \vee D_{i}\right) \cap T$ is nonvoid by Lemma 2.5. It follows that $H \cap T$ is nonvoid. Hence $H$ is a maximal antichain. Since $H \subset C \vee D$, 
$H=C \vee D$ and $\vee$ is a continuous operation on $\mathscr{A}(X)$. Similarly $\wedge$ is a continuous operation on $\mathscr{A}(X)$.

If $X$ is metric then by Theorem 2.6 there exists an arc in $\mathscr{A}(X)$ which contains the maximal and minimal elements of $\mathscr{A}(X)$. It follows that $\mathscr{A}(X)$ is arcwise connected.

\section{A characterization of the 2-cell.}

THEOREM 5.1. Let $X$ be a nondegenerate metric partially ordered space. Suppose there exists a map $f:[0,1] \rightarrow 2^{X}$ such that

(i) for each $a \in[0,1], f(a) \in \mathscr{M}(X)$,

(ii) if $a \leqq b \leqq c$ in $[0,1]$ then $f(a) \cap f(c) \subset f(b)$ and

(iii) $X \subset \cup f([0,1])$.

If $X$ has no cutpoints then $X$ is homeomorphic to the closed 2-cell.

Proof. It is well known (see Franklin [9]) that the relation $R:[0,1] \rightarrow X$ defined by letting $R(a)=f(a)$ for each $a \in[0,1]$ is upper semicontinuous. Since $R(a)$ is compact and connected for each $a \in[0,1], X=R([0,1])$ is compact and connected by [9].

If $a \in[0,1]$ such that $f(0) \neq f(a) \neq f(1)$ then $f(a)$ separates $X$ since $f([0, a])$ $\cap f([a, 1])=f(a)$. Since $X$ has no cutpoints it follows that if $f(a)$ is a point then either $f(a)=f(0)$ or $f(a)=f(1)$.

The set $\{f(a) \mid a \in[0,1]\}$ is a compact family of maximal chains of $X$ such that $X \subset \bigcup\{f(a) \mid a \in[0,1]\}$. By Lemma 1.4, $L$ and $M$ are compact.

Consider the function $\pi:[0,1] \rightarrow M$ defined by letting $\pi(a) \in f(a) \cap M$ for each $a \in[0,1]$. Let $\left.a_{i}\right)$ be a net in $[0,1]$ which converges to $a$. For each $i, f\left(a_{i}\right) \subset L\left(\pi\left(a_{i}\right)\right)$. If $z$ is a cluster point of $\pi\left(a_{i}\right)$ ) then $f(a) \subset L(z)$ since the partial order on $X$ is closed. Now $\pi(a) \in f(a)$ so $\pi(a) \in L(z)$. Since $\pi(a) \in M$ it follows that $z=\pi(a)$ and $\pi$ is continuous. By condition (ii) of the hypotheses $\pi$ is monotone. Hence, $M$ is either a point or an arc. Similarly, $L$ is either a point or an arc. Thus, $f(0) \cup f(1) \cup M \cup L$ is a simple closed curve.

Suppose $L \cap M$ is empty. By Theorem 2.9 there exists a map $g: P([0,1]) \rightarrow X$ such that for each $a \in[0,1], g$ maps $\{a\} \times[0,1]$ homeomorphically onto $f(a)$.

For each $x \in X, g^{-1}(x)$ is a compact antichain. Also, for each $x \in X$

$$
\{a \in[0,1] \mid x \in f(a)\}
$$

is connected by condition (ii) of the hypotheses. It is now easy to check that $g$ is monotone. By a Theorem of R. L. Moore (Whyburn [8, p. 173]) $X$ is a 2-cell.

If $L \cap M$ is nonvoid then we must go back to the methods used in Case 2 of Theorem 2.6 to obtain a monotone map of $P([0,1])$ onto $X$. The argument is completely straightforward.

COROllary 5.2. Let $X$ be a nondegenerate compact metric space with no cutpoints. Then $X$ is a closed 2-cell if and only if $X$ admits a closed partial order such that 
(i) $M$ is an arc,

(ii) $L$ is closed,

(iii) for each $m \in M, L(m)$ is an order arc,

(iv) $L(m)$ separates $X$ for each cutpoint $m$ of $M$.

Proof. The partially ordered space $P([0,1])$ is a partially ordered space on the 2-cell which satisfies all the hypotheses of the theorem.

If $X$ has a partial order satisfying the hypotheses of the theorem let $h$ be a homeomorphism of $[0,1]$ onto $M$ and define $f:[0,1] \rightarrow 2^{x}$ by letting $f(a)=L(h(a))$ for each $a \in[0,1]$. By Theorem 3.2, $f$ is continuous. Condition (iv) of the hypotheses implies that if $a \leqq b \leqq c$ in $[0,1]$ then $f(a) \cap f(c) \subset f(b)$. Hence Theorem 5.1 applies.

\title{
REFERENCES
}

1. J. H. Carruth, A note on partially ordered compacta, Pacific J. Math. 24 (1968), 229-231. MR 36 \#5902.

2. J. L. Kelley, General topology, Van Nostrand, Princeton, N. J., 1955. MR 16, 1136.

3. E. Michael, Topologies on spaces of subsets, Trans. Amer. Math. Soc. 71 (1951), 152-182. MR 13, 54.

4. L. Nachbin, Topology and order, Van Nostrand, Princeton, N. J., 1965. MR 36 \#2125.

5. L. E. Ward, Jr., Partially ordered topological spaces, Proc. Amer. Math. Soc. 5 (1954), 144-161. MR 16, 59.

6. - Concerning Koch's theorem on the existence of arcs, Pacific J. Math. 15 (1965), 347-355. MR 31 \#6206.

7. L. E. Ward, Jr. and E. D. Tymchatyn, On three problems of S. P. Franklin and A. D. Wallace, Colloq. Math. 20 (1969), 229-236.

8. G. T. Whyburn, Analytic topology, Amer. Math. Soc. Colloq. Publ., vol. 28, Amer. Math. Soc., Providence, R. I., 1942. MR 4, 86.

9. S. P. Franklin, Concerning continuous relations, Dissertation, University of California, Los Angeles, Calif., 1963.

\author{
UNIVERSITY OF SASKaTChEWAN, \\ Saskatoon, Canada \\ UNIVERSITY OF OREGON, \\ Eugene, Oregon
}

